\title{
The Jovian Rings
}

\author{
Wing-Huen Ip \\ Institute of Astronomy \\ National Central University, Taiwan \\ email: wingip@astro.ncu.edu.tw
}

\begin{abstract}
A comparison of the Jovian and Saturnian rings is made by reviewing the recent advances in planetary spacecraft exploration and theoretical study. Two main issues are addressed, namely, the different structures of these two planetary ring systems and the water ice composition of the Saturnian rings. It is suggested that answers might be found by invoking tidal capture of Trans-Neptunian Objects with highly differentiated structures even though catastrophic breakup of pre-existing satellites in the ring regions remains a real possibility. Erosion mechanisms such as meteoroid impact, photo-sputtering, orbital instability of charged dust particles and thermal evaporation acting at different time scales could lead to the preservation of the Saturnian ring system but not the Jovian ring system of large mass originally.
\end{abstract}

Keywords. Jupiter, the Jovian rings, the Saturnian rings, erosion, gardening.

\section{Introduction}

At this conference, we celebrated the 400th anniversary of Galileo's discovery of the four Medicean moons of Jupiter in early January 1610. Because of the important philosophical and intellectual impacts on the world view by this unique observational result, it has been generally regarded as the apex of Galileo's fundamental contribution to astronomy and science. His other exciting discovery, namely, that of the Saturnian rings in the middle of July of the same year was generally not well publicized. In fact, that Saturn was observed to be not round and single but rather triple must have come as a real surprise to Galileo (van Helden, 1984a, 1984b). This puzzle was finally solved by Christian Huygens (16291695) who showed that Saturn was surrounded by a flat disk. Another breakthrough came when Giovanni Domenico Cassini (1625-1712) detected the existence of a gap dividing the disk into two parts which was later named after him. Ground-based study of the Saturnian rings was pursued until the very eve of the first close-up imaging observations by the Voyager 1 spacecraft (Dollfus, 1979a, b). Before the flyby of the Saturnian rings of Voyager 1 the monopoly of Saturn in owning a ring system was already broken by the serendipitous discovery of a system of narrow rings around Uranus by Elliot et al. (1977). The same technique of ground-based occultation measurements was used in the early 80's of the 20th Century to detect Neptune's narrow rings and a series of ring arcs (Manfroid et al., 1986; Hubbard, 1986). For Jupiter, the crowning moment came when Voyager 1 obtained a most exquisite image of the Jovian rings (see Figure 1). Since then a lot of important observations and theoretical studies have been dedicated to understand the structure and dynamics of the Jovian rings which are mainly composed of small dust particles (see review by Burns et al. (2004) and references therein). The Jovian ring detected by Voyager 1 has an outer edge at $1.81 \mathrm{R}_{J}$ (Jovian radii) which is also the orbital distances of two tiny satellites, J15 Adrastea and J16 Metis. Recent observations have shown clearly that the main ring detected by Voyager 1 is at the inner edge of yet another broad, tenuous disk of dust extending all the way to $3.1 \mathrm{R}_{J}$. The material can be traced to two small satellites, Amalthea and Thebe (Showalter et al., 2008). The 
formation of the tenuous Jovian rings as a result of ejection of surface material from some parent bodies has interesting counter part in the Saturnian rings. That is, the G ring, the Janus/Epimetheus ring and thePallene ring as shown in Figure 2, are in principle very similar to the Jovian rings if the main Saturn rings inside the orbit of Janus could be taken away.

If Galileo had been able to see these different ring systems, the first question he would ask would probably be why only Saturn has a sizable ring system and not others? To answer this fundamental question, we will argue in the following that a comparison of the similarities and dissimilarities of the Jovian and Saturnian rings might give us some hints.

\section{Thick Disk vs. Narrow Rings}

In comparison with the Jovian rings with an average optical depth (tau) of $\sim$ a few $10^{-6}$ the rings of Saturn is much thicker and more massive. The total mass can be estimated to be on the order of $7.0 \times 10^{22} \mathrm{~g}$ assuming a water ice composition. If all the mass is collected into a single body, it would be of the size (diameter) of $225 \mathrm{~km}$. It is very unlikely that this ring system is of primordial origin. This is because the inner region of the satellitary nebula surrounding Saturn should have a temperature on the order of $1000 \mathrm{~K}$ during formation of the planetary satellites (Ayliffe and Bate, 2009), and such a high temperature would not allow the co-accretion of Saturn and the icy ring system. The breakup of an inner satellite due to catastrophic impact with a large projectile has been postulated to be a possible origin of the ring system (Pollack, 1975; Ip, 1988). An alternative possibility is to invoke the tidal disruption of one or more large comets during their close passages inside Saturn's Roche limit (Dones, 1991). Charnoz et al. (2009) revisited this issue and reached the conclusion that either mechanism is a possible origin of the Saturnian rings in spite of some intrinsic difficulties of each process. According to their dynamical study, the ring formation event might have taken place during the so-called Late Heavy Bombardment Event (LHB) at which time ( $~ 3.8$ billion years ago) an intense flux of TNOs should have been injected into Saturn-crossing orbits from outside. There is, however, an important conundrum concerning the lifetime of the Saturn rings. That is, a number of studies have shown that the Saturn rings are subject to

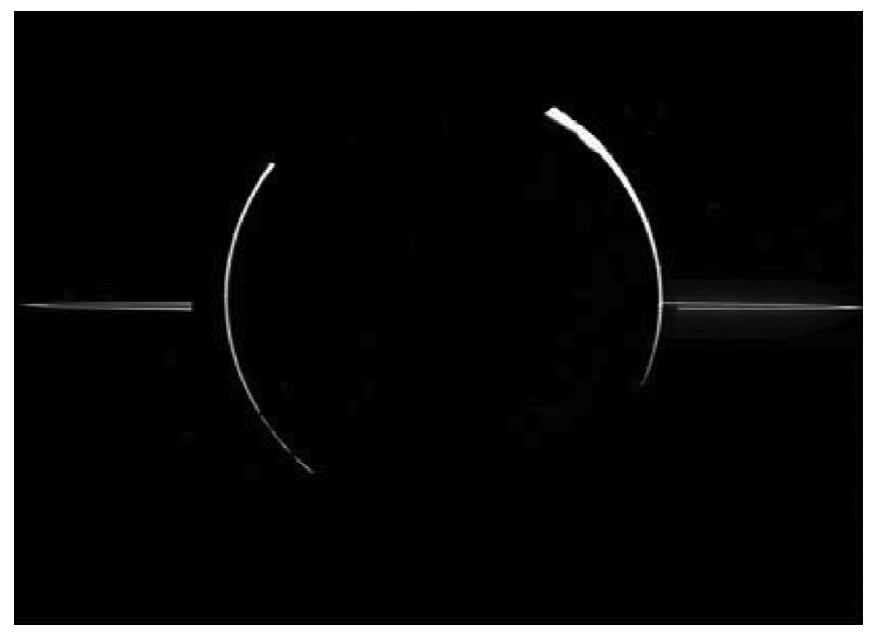

Figure 1. The Jovian rings from the imaging observations of Voyager 1. From NASA JPL. 
rapid erosion effects as a consequence of orbital decay, interplanetary meteoroid impact, plasma siphon flow and photosputtering (Morfill et al., 1980; Ip, 1983, 2005; Johnson and Quickenden, 1997; Johnson et al., 2006). The estimates of the ring lifetime have been given to be between 200 million years and one billion years (Morfill et al., 1983; Ip, 1989; Farmer and Goldreich, 2007). Barring the event that these parameter studies are wrong by orders of magnitude, it is reasonable to cast in doubt the primordial nature of the Saturnian rings. In this event, the existing ring material must have been replenished since LHB. This could be achieved by continuous impact capture of incoming TNOs (Trans-Neptunian Objects) over the eons.

In the model calculations of Charnoz et al. (2009), the difficulties of both tidal disruption of TNOs and satellite breakup mechanism were discussed. The problem posed by the exogenous origin has to do with the fact that among the outer planets, Jupiter had the biggest chance to acquire a ring system via tidal disruption/capture of TNOs while Saturn, Uranus and Neptune had a much smaller probability of doing so. For the satellite origin, several inner icy satellites (like Janus and Mimas) of Saturn should have been destroyed during the episode of intense bombardment according to this mechanism. However, these small satellites could have reformed via re-accretion since they are outside the Roche limit.

Assuming that Jupiter had a thick ring system like that of Saturn immediately after LHB - whatever the production mechanism, its disappearance could be understood in terms of the much faster erosive effects by a factor of 4-5 (i.e., thermal sublimation, photosputtering, meteoroid impact and orbital instability of the charged small grains) mentioned earlier because of its closer distance to the Sun. Amalthea which has a density as small as $0.86 \mathrm{~g} \mathrm{~cm}^{-3}$ (Anderson et al., 2005) could be the remnant of such a process. It could have been pushed outward because of the tidal effect. The same can probably be said of Janus in the case of the Saturnian ring system.

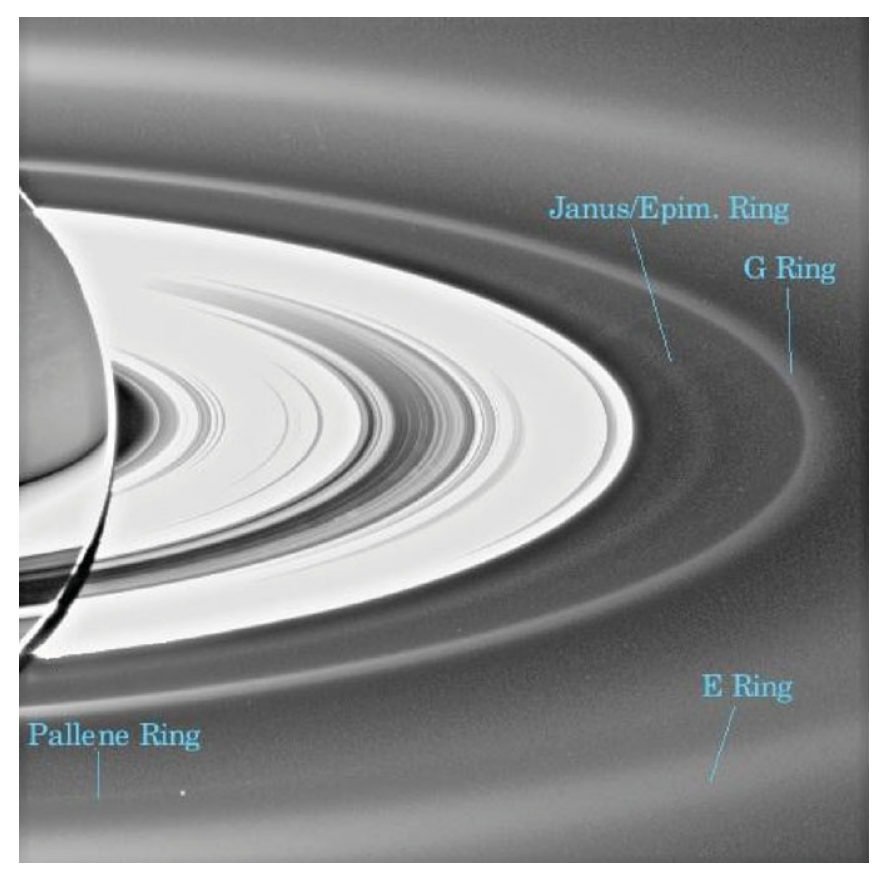

Figure 2. An image from the Cassini mission showing the Saturnian rings with the faint rings associated with small satellites indicated. From NASA JPL. 


\section{Gardening on the Rings}

The remaining issue has to do with the composition of the Saturn rings which are mainly water ice. The average densities of large TNOs and Enceladus are comparable and of the order of $1.5-2 \mathrm{~g} \mathrm{~cm}^{-3}$ basically suggesting that they must contain a significant amount of rocky materials. We must then accept the scenario that the original ring composition should be a mixture of ice and silicate material at the very beginning. The question is how to hide the rocky part from view. Where has it gone? Or rather where has all the water ice come from?

Recent Cassini imaging observations of the small Saturnian moon, S12 Helene, which is orbiting at $6.25 \mathrm{R}_{S}$ (Saturnian radii) showed that its very smooth surface might have been shaped by the deposit of icy grains from the E ring. Can similar mechanism be operational at the ring system? Noting that the present-day production rate of $\mathrm{H}_{2} \mathrm{O}$ molecules from Enceladus is of the order of $10^{28}$ molecules $\mathrm{s}^{-1}$. (Waite et al., 2006; Richardson and Jurac, 2004 ), the total mass emitted over a time interval of $10^{7}$ years would be $10^{20} \mathrm{~g}$ assuming that the outgassing rate could be kept constant. With about $10 \%$ of the E ring material being intercepted by the outer edge of the ring system (Jurac and Richardson, 2007), the accreted mass would be on the order of $10^{19} \mathrm{~g}$ which is comparable to the mass of a small satellite with a radius of $13 \mathrm{~km}$. Even though this mass is much smaller than that of the ring mass, it could change the outlook of the ring system completely if the volatile ice can be added to the top layer of the ring particles thus disguising their true nature.

Another mechanism is to invoke recycling of the icy material of the original population of the broken pieces produced by tidal disruption of a TNO or catastrophic fragmentation of a satellite. The surfaces of planetary bodies like asteroids and planetary satellites are subject to collisional bombardment by interplanetary meteoroids and dust particles. Such hypervelocity impact would produce craters and eject surface materials to different regions depending on the emission speed. By the same token, the surface layer up to a certain depth (i.e., the regolith layer) is made up of fragments coming from various parts of the object. This process is called surface "gardening". It is expected that similar mechanism should take place in the Saturnian rings and that the ring particles would be covered by a layer of broken pieces. But in the context of the ring system, some new effect has to be taken into consideration. That is, as described before, the volatile water ice on the surface is constantly subject to erosion and redistribution effect as a result of solar ultraviolet radiation and meteoroid impact vaporization. As a result, most if not all of the ring particles would be covered by a layer of water ice even if they are originally of rocky composition. In other words, we expect that the ring particles - large or small - should have a two-layer structure even though they were not subject to any thermal differentiation effect as in the case of large icy satellites. In this manner, the rings can be painted white.

\section{Summary}

In the above we have discussed how impact gardening and ballistic transport of the volatile water across the ring plane could lead to the appearance of water ice composition of the Saturnian ring particles. In addition, we have also suggested that the absence of a thick ring around Jupiter could be the result of faster erosion than that of the Saturnian rings. This argument depends a lot on the volatility of the icy material in the rings. If a significant part of the ring material (embedded inside the ice matrices) is of rocky composition, the existence of a disk of rocky particles should remain since 
thermal evaporation and photosputtering would be very ineffective in eroding them. We are therefore returning to the same question asked by Charnoz et al. (2009) on the original composition of the Saturnian rings and that of the putative Jovian rings of much larger mass than found in the present system. One way out - as suggested by these authors is perhaps to invoke tidal capture of the outer shells of thermally differentiated TNOs while leaving the inner core of rocky and metallic cores continued on their heliocentric orbits. A corollary of this scenario is that J15 Adrastea and J16 Metis, which define the outer edge of the main Jovian ring, would have relatively large density $\left(\rho \sim 3-4 \mathrm{~g} \mathrm{~cm}^{-3}\right)$ since they might represent the remnants of the inner core material of the captured TNOs.

Acknowledgment I thank the organizers of this IAU symposium, especially Professor Cesare Barbieri, for invitation and financial support. This work was partly supported by NSC Grant: NSC 96-2752-M-008-011-PAE and Ministry of Education under the Aim for Top University Program NCU.

\section{References}

Anderson, J. D., Johnson, T. V., Schubert, G., Asmar, S., Jacobson, R. A., Johnston, D., Lau, E. L., Lewis, G., Moore, W. B., Taylor, A., Thomas, P. C., \& Weinwurn, G. 2005, Science, 308,1291

Ayliffe, B. A. \& Bate, M. R. 2009, Mon. Not. R. Astron. Soc, 397, 657

Burns, J. A., Simonelli, D. P., Showalter, M. R., Hamilton, D. P., Proco, C. C., Throop, H., \& Esposito, L. W. 2004, in Jupiter, F. Bagenal, T. Dowling, \& W. McKinnon (eds.), (Cambridge University Press), p.241

Dollfus, A. 1979a, Icarus, 37, 404

Dollfus, A. 1979b, Icarus, 40, 171

Elliot, J. L., Dunham, E. W., \& Mink, D. J. 1977, Nature, 267, 328

Farmer, A. J. \& Goldreich, P. 2007, Icarus, 188, 108

Hubbard, W. B., Brahic, A., Sicardy, B., Elicer, L. R., Roques, F., \& Vilas, F. 1986, Nature, 319,636

Ip, W.-H. 1983, J. Geophys. Res., 88, 819

Ip, W.-H. 1984, Icarus, 60, 547

Ip, W.-H. 1988, A\& A, 199, 340

Ip, W.-H. 2005, Geophys. Res. Lett., 32, Issue 13, CiteID L 13204

Johnson, R. E. \& Quickenden, T. I. 1997, J. Geophys. Res., 102, E5, 10985

Johnson, R. E., Luhmann, J. G., Tokar, R. C., Bouhram, M., Berthelier, J., Sittler, E. C., Cooper, J. F., Hill, T. W., Michael, M., Liu, J., Crary, F. J., \& Young, D. T. 2006, Icarus, 180,393

Jurac, S. \& Richardson, J. D. 2007, Geophys. Res. Lett., 34, Issue 8, CitreID L08102

Manfroid, M. S., Haefner, R., \& Bouchet, P. 1986, A\& A, 157, L3

Morfill, G. E., Fechtig, H., Gruen, E., \& Goertz, C. K. 1983, Icarus, 55, 439

Richardson, J. E. \& Jurac, S. 2004, Geophys. Res. Lett., 31, Issue 24, CiteIDL24803

Showalter, W. R., de Pater, I., Verbanac, G., Hamilton, D. P., \& Burns, J. A 2008, Icarus, 195, 361

Van Helden, A. 1984a, in R. Greenberg \& A. Brahic (Eds.), Plantary Rings, (University of Arizona Press), p. 12

Van Helden, A. 1984b, in T. Gehrels \& M.S. Matthews (Eds.), Saturn, (University of Arizona Press), p. 23

Waite, J. H., Combi, M. R., Ip, W.-H., Cravens, T. E., McNutt, R. L., Kasprzak, W., Yelle, R., Luhmann, J., Niemann, H., Gell, D., Magee, B., Fletcher, G., Lunine, J., \& Tseng, W.-L. 2006, Science, 311, 1419 